\title{
Improving Physicians' Bowel Documentation on Geriatric Wards
}

\author{
Alexander P. Noar, BMBCh, BA, Jessica Parkin, MBChB, BSc, Tharindri Wijekoon, MBChB, \\ PGCert, Rusyai Zalynda, MBChB, Jennifer Tsui, MBChB, and Kokwai Chin, MBBS, MRes
}

Objective: Constipation is widely prevalent in older adults and may result in complications such as urinary retention, delirium, and bowel obstruction. Previous studies have indicated that while the nursing staff do well in completing stool charts, doctors monitor them infrequently. This project aimed to improve the documentation of bowel movement by doctors on ward rounds to $85 \%$, by the end of a 3-month period.

Methods: Baseline, postintervention, and sustainability data were collected from inpatient notes on weekdays on a geriatric ward in Northern General Hospital, Sheffield, UK. Posters and stickers of the poo emoji were placed on walls and in inpatient notes, respectively, as a reminder.

Results: Data on bowel activity documentation were collected from 28 patients. The baseline data showed that bowel activity was monitored daily on the ward $60.49 \%$ of the time. However, following the interventions, there was a significant increase in documentation, to $86.78 \%$. The sustainability study showed that bowel activity was documented on the ward $56.56 \%$ of the time.

Conclusion: This study shows how a strong initial effect on behavioral change can be accomplished through simple interventions such as stickers and posters. As most wards currently still use paper notes, this is a generalizable model that other wards can trial. However, this study also shows the difficulty in maintaining behavioral change over extended periods of time.

Keywords: bowel movement; documentation; obstruction; constipation; geriatrics; incontinence; junior doctor; quality improvement.

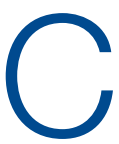
onstipation is widely prevalent in the elderly, encountered frequently in both community and hospital medicine. ${ }^{1}$ Its estimated prevalence in adults over 84 years old is $34 \%$ for women and $25 \%$ for men, rising to up to $80 \%$ for long-term care residents. ${ }^{2}$

Chronic constipation is generally characterized by unsatisfactory defecation due to infrequent bowel emptying or difficulty with stool passage, which may lead to incomplete evacuation. ${ }^{2-4}$ Constipation in the elderly, in addition to causing abdominal pain, nausea, and reduced appetite, may result in complications such as fecal incontinence (and overflow diarrhea), urinary retention, delirium, and bowel obstruction, which may in result in life-threatening perforation.,.$^{5,6}$ For inpatients on geriatric wards, these consequences may increase morbidity and mortality, while prolonging hospital stays, thereby also increasing exposure to hospital-acquired infections. ${ }^{7}$ Furthermore, constipation is also associated with impaired health-related quality of life. ${ }^{8}$

Management includes treating the cause, stopping contributing medications, early mobilization, diet modification, and, if all else fails, prescription laxatives. Therefore, early identification and appropriate treatment of constipation is beneficial in inpatient care, as well as when planning safe and patient-centered discharges.

Given the risks and complications of constipation in the elderly, we, a group of Foundation Year 2 (FY2) doctors in the UK Foundation Programme, decided to explore how doctors can help to recognize this condition early. Regular bowel movement documentation in patient notes on ward rounds is crucial, as it has been shown to reduce constipation-associated complications. ${ }^{5}$ However, complications from constipation can

From Sheffield Teaching Hospitals, Sheffield, UK, S5 7AU. 


\begin{tabular}{llll}
\hline \multicolumn{2}{l}{ Table. Patient Encounters Preintervention, Postintervention, and 1-Month Postintervention (Sustainability) } \\
\hline & & \multicolumn{2}{c}{ Total number of patient encounters } \\
\hline Patient group & Preintervention & Postintervention & Sustainability phase \\
\hline Screened & 420 & 280 & 280 \\
\hline Excluded & 129 & 47 & 43 \\
& & End of life $(n=29)$ & End of life $(n=15)$ \\
& & Discharged $(n=10)$ & Discharged $(n=13)$ \\
Incomplete $(n=15)$
\end{tabular}

take significant amounts of time to develop and, therefore, documenting bowel movements on a daily basis is not necessary.

Based on these observations along with targets set out in previous studies, ${ }^{7}$ our aim was to improve documentation of bowel movement on ward rounds to $85 \%$ by March 2020.

\section{Methods}

Before the data collection process, a fishbone diagram was designed to identify the potential causes of poor documentation of bowel movement on geriatric wards. There were several aspects that were reviewed, including, for example, patients, health care professionals, organizational policies, procedures, and equipment. It was then decided to focus on raising awareness of the documentation of bowel movement by doctors specifically.

Retrospective data were collected from the inpatient paper notes of 28 patients on Brearley 6, a geriatric ward at the Northern General Hospital within Sheffield Teaching Hospitals (STH), on weekdays over a 3-week period. The baseline data collected included the bed number of the patient, whether or not bowel movement on initial ward round was documented, and whether it was the junior, registrar, or consultant leading the ward round. End-of-life and discharged patients were excluded (Table).

The interventions consisted of posters and stickers. Posters were displayed on Brearley 6 , including the doctors' office, nurses' station, and around the bays where notes were kept, in order to emphasize their importance. The stickers of the poo emoji were also printed and placed at the front of each set of inpatient paper notes as a reminder for the doctor documenting on the ward round. The interventions were also introduced in the morning board meeting to ensure all staff on Brearley 6 were aware of them.

Data were collected on weekdays over a 3-week period starting 2 weeks after the interventions were put in place (Table). In order to assess that the intervention had been sustained, data were again collected 1 month later over a 2-week period (Table). Microsoft Excel (Microsoft Corporation, Redmond, Washington, USA) was used to analyze all data, and control charts were used to assess variability in the data.

\section{Results}

The baseline data showed that bowel movement was documented $60.49 \%$ of the time by doctors on the initial ward round before intervention, as illustrated in Figure 1. There was no evidence of an out-of-control process in this baseline data set.

The comparison between the preintervention and postintervention data is illustrated in Figure 1. The postintervention data, which were taken 2 weeks after intervention, showed a significant increase in the documentation of bowel movements, to $86.78 \%$. The figure displays a number of features consistent with an out-of-control process: beyond limits ( $\geq 1$ points beyond control limits), Zone A rule (2 out of 3 consecutive points beyond 2 standard deviations from the mean), Zone $\mathrm{B}$ rule (4 out of 5 consecutive points beyond 1 standard deviation from the mean), and Zone $C$ rule ( $\geq 8$ consecutive points on 1 side of the mean). These findings demonstrate a special cause variation in the documentation of bowel movements. 


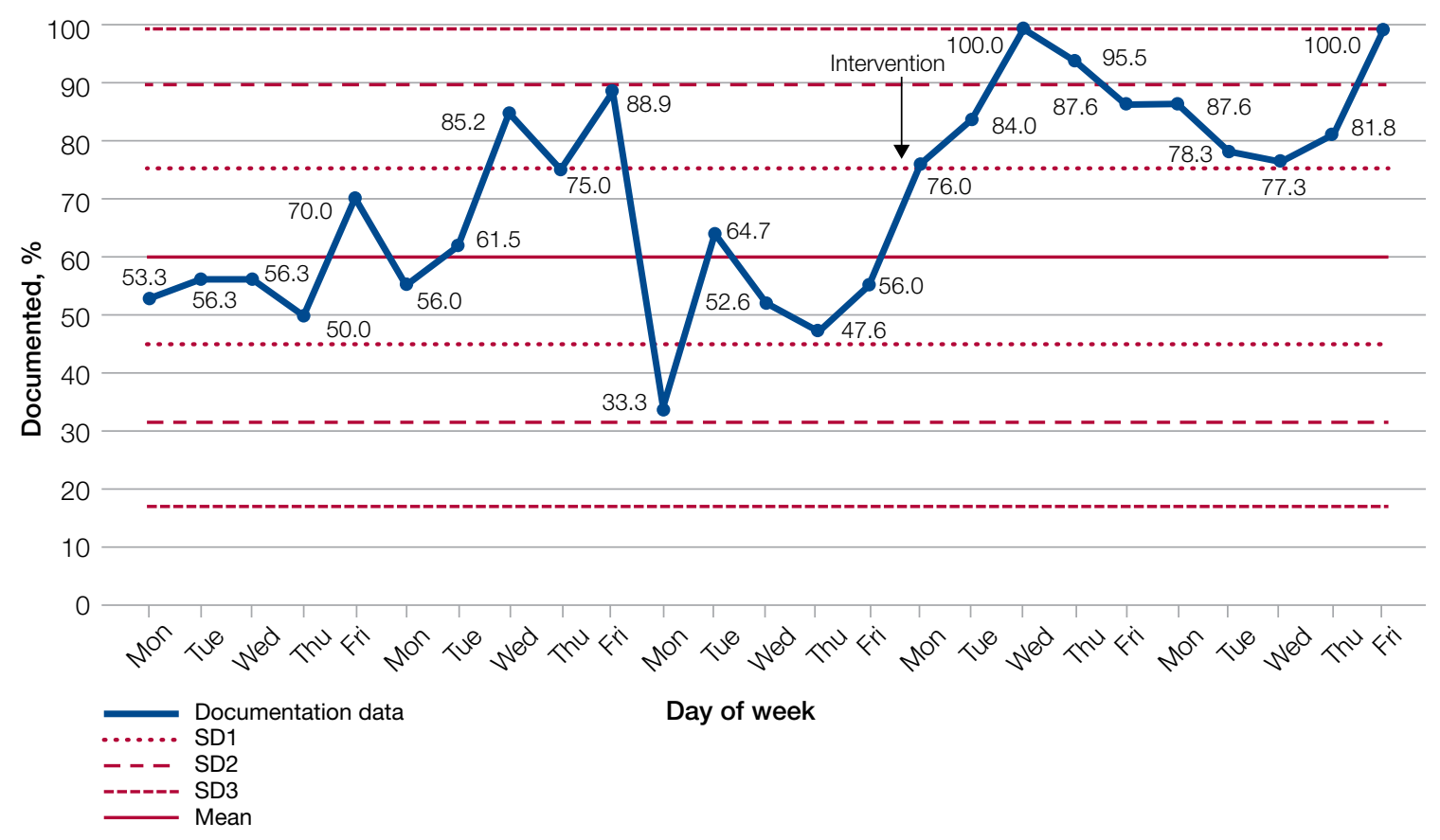

Figure 1. Bowel movement documentation data preintervention and postintervention including mean and standard deviations (SDs).

Figure 2 shows the sustainability of the intervention, which averaged $56.56 \%$ postintervention nearly 2 months later. The data returned to preintervention variability levels.

\section{Discussion}

Our project explored an important issue that was frequently encountered by department clinicians. Our team of FY2 doctors, in general, had little experience with quality improvement. We have developed our understanding and experience through planning, making, and measuring improvement.

It was challenging deciding on how to deal with the problem. A number of ways were considered to improve the paper rounding chart, but the nursing team had already planned to make changes to it. Bowel activity is mainly documented by nursing staff, but there was no specific protocol for recognizing constipation and when to inform the medical team. We decided to focus on doctors' documentation in patient notes during the ward round, as this is where the decision regarding management of bowels is made, including interventions that could only be done by doctors, such as prescribing laxatives.
Strom et $\mathrm{al}^{9}$ have described a number of successful quality improvement interventions, and we decided to follow the authors' guidance to implement a reminder system strategy using both posters and stickers to prompt doctors to document bowel activity. Both of these were simple, and the text on the poster was concise. The only cost incurred on the project was from printing the stickers; this totalled £2.99 (US \$4.13). Individual stickers for each ward round entry were considered but not used, as it would create an additional task for doctors.

The data initially indicated that the interventions had their desired effect. However, this positive change was unsustainable, most likely suggesting that the novelty of the stickers and posters wore off at some point, leading to junior doctors no longer noticing them. Further PlanDo-Study-Act cycles should examine the reasons why the change is difficult to sustain and implement new policies that aim to overcome them.

There were a number of limitations to this study. A patient could be discharged before data collection, which was done twice weekly. This could have resulted in 


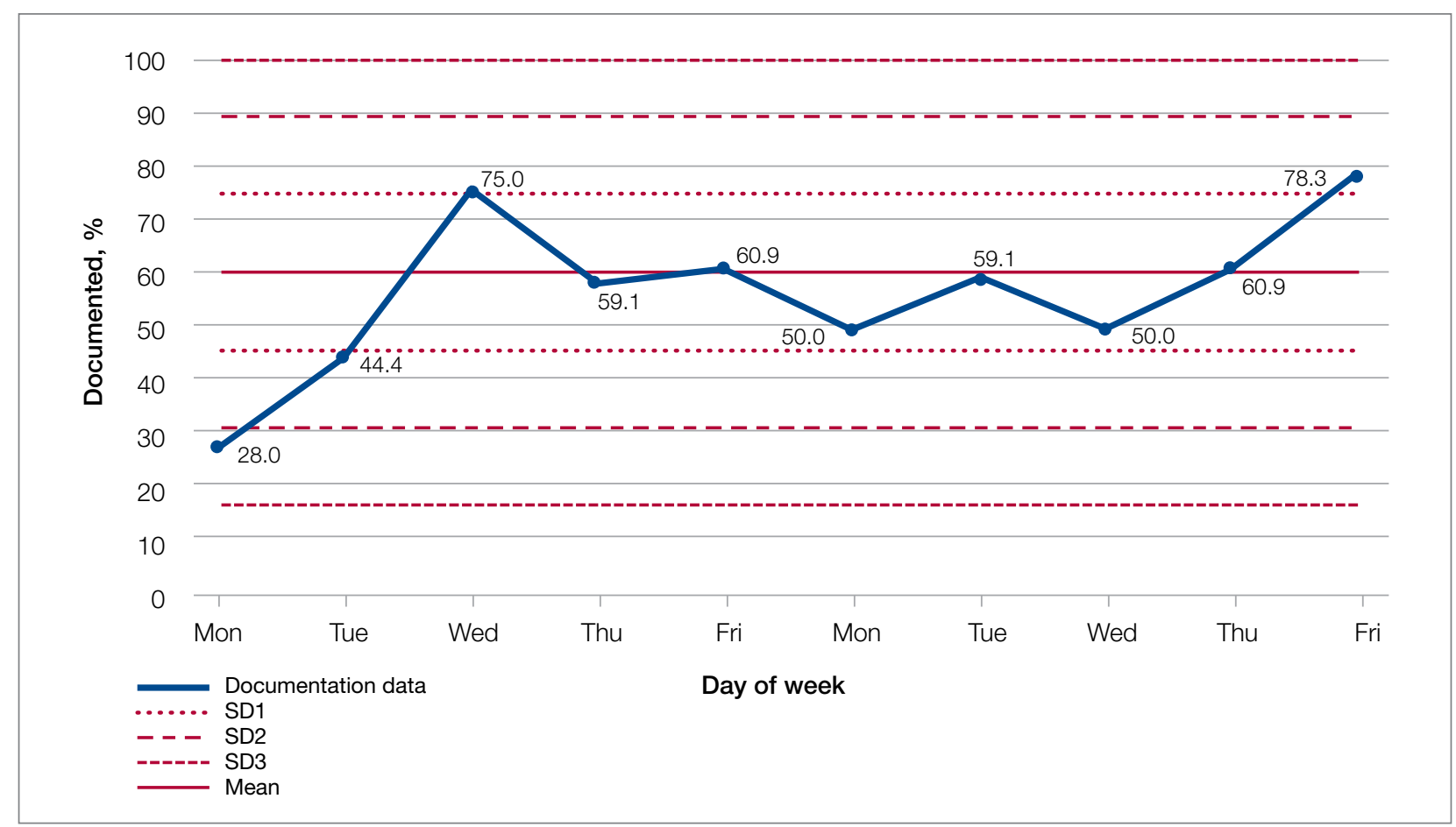

Figure 2. Sustainability data 2 months postintervention including mean and standard deviations (SDs).

missed data during the collection period. In addition, the accuracy of the documentation is dependent on nursing staff correctly recording-as well as the doctors correctly viewing-all sources of information on bowel activity. Observer bias is possible, too, as a steering group member was involved in data collection. Their awareness of the project could cause a positive skew in the data. And, unfortunately, the project came to an abrupt end because of COVID-19 cases on the ward.

We examined the daily documentation of bowel activity, which may not be necessary considering that internationally recognized constipation classifications, such as the Rome III criteria, define constipation as fewer than 3 bowel movements per week. ${ }^{10}$ However, the data collection sheet did not include patient identifiers, so it was impossible to determine whether bowel activity had been documented 3 or more times per week for each patient. This is important because a clinician may only decide to act if there is no bowel movement activity for 3 or more days.

Because our data were collected on a single geriatric ward, which had an emphasis on Parkinson's disease, it is unclear whether our findings are generalizable to other clinical areas in STH. However, constipation is common in the elderly, so it is likely to be relevant to other wards, as more than a third of STH hospital beds are occupied by patients aged 75 years and older. ${ }^{11}$

\section{Conclusion}

Overall, our study highlights the fact that monitoring bowel activity is important on a geriatric ward. Recognizing constipation early prevents complications and delays to discharge. As mentioned earlier, our aim was achieved initially but not sustained. Therefore, future development should focus on sustainability. For example, laxative-focused ward rounds have shown to be effective at recognizing and preventing constipation by intervening early. ${ }^{12}$ Future cycles that we considered included using an electronic reminder on the hospital IT system, as the trust is aiming to introduce electronic documentation. Focus could also be placed on improving documentation in bowel charts by ward staff. This could be achieved by organizing regular educational sessions on the complications of constipation and when to inform the medical team regarding concerns. 
Acknowledgments: The authors thank Dr. Jamie Kapur, Sheffield Teaching Hospitals, for his guidance and supervision, as well as our collaborators: Rachel Hallam, Claire Walker, Monisha Chakravorty, and Hamza Khan.

Corresponding author: Alexander P. Noar, BMBCh, BA, 10 Stanhope Gardens, London, N6 5TS; alecnoar@live.co.uk.

Financial disclosures: None.

doi:10.12788/jcom.0063

\section{References}

1. Forootan M, Bagheri N, Darvishi M. Chronic constipation: A review of literature. Medicine (Baltimore). 2018;97:e10631. doi:10.1097/ MD.00000000000.10631

2. Schuster BG, Kosar L, Kamrul R. Constipation in older adults: stepwise approach to keep things moving. Can Fam Physician. 2015;61:152-158.

3. Gray JR. What is chronic constipation? Definition and diagnosis. Can J Gastroenterol. 2011;25 (Suppl B):7B-10B.

4. American Gastroenterological Association, Bharucha AE, Dorn SD, Lembo A, Pressman A. American Gastroenterological Association medical position statement on constipation. Gastroenterology. 2013;144:211-217. doi:10.1053/j.gastro.2012.10.029
5. Maung TZ, Singh K. Regular monitoring with stool chart prevents constipation, urinary retention and delirium in elderly patients: an audit leading to clinical effectiveness, efficiency and patient centredness. Future Healthc J. 2019;6(Suppl 2):3. doi:10.7861/futurehosp.6-2s-s3

6. Mostafa SM, Bhandari S, Ritchie G, et al. Constipation and its implications in the critically ill patient. Br J Anaesth. 2003;91:815-819. doi:10.1093/bja/aeg275

7. Jackson R, Cheng P, Moreman S, et al. "The constipation conundrum": Improving recognition of constipation on a gastroenterology ward. BMJ Qual Improv Rep. 2016;5(1):u212167.w3007. doi:10.1136/bmjquality.u212167.w3007

8. Rao S, Go JT. Update on the management of constipation in the elderly: new treatment options. Clin Interv Aging. 2010;5:163-171. doi: $10.2147 /$ cia.s8100

9. Strom KL. Quality improvement interventions: what works? J Healthc Qual. 2001;23(5):4-24. doi:10.1111/j.1945-1474.2001.tb00368.x

10. De Giorgio R, Ruggeri E, Stanghellini V, et al. Chronic constipation in the elderly: a primer for the gastroenterologist. BMC Gastroenterol. 2015;15:130. doi:10.1186/s12876-015-366-3

11. The Health Foundation. Improving the flow of older people. April 2013. Accessed August 11, 2021. https://www.england.nhs.uk/ wp-content/uploads/2013/08/sheff-study.pdf

12. Linton A. Improving management of constipation in an inpatient setting using a care bundle. BMJ Qual Improv Rep. 2014;3(1):u201903. w1002. doi:10.1136/bmjquality.u201903.w1002 